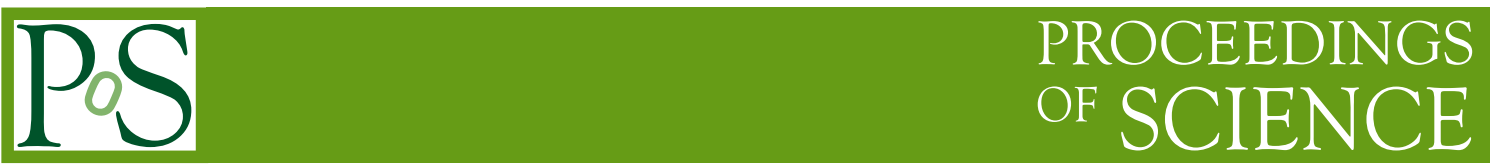

\title{
JUNO Oscillation Physics Program
}

\section{Gioacchino Ranucci ${ }^{1}$ on behalf of the JUNO Collaboration}

Istituto Nazionale di Fisica Nucleare

Via Celoria 16, 20133 Milano, Italy

E-mail: gioacchino . ranucci@mi . infn. it

The JUNO (Jiangmen Underground Neutrino Observatory), a 20 kton multi-purpose underground liquid scintillator detector, has been proposed and approved for realization in the south of China. After an intense design phase, the overall concept of the structure of the detector has been finalized, paving the way towards the construction of the several components and subsystems, which will compose it. Meanwhile, the excavation of the site, which will host the experiment has been started and is rapidly progressing. The main physics target of JUNO is the determination of the neutrino mass hierarchy, which will be accessible through the measurement of the antineutrino spectrum from two high power nuclear complexes under installation $53 \mathrm{~km}$ away from the experimental site. In this work, I describe the deep physics capabilities of the experiment in term of neutrino oscillation physics, which include in addition to the crucial measure of the neutrino hierarchy the high precision determination of three oscillation parameters, and I illustrate the main technical characteristics of the detector.

Neutrino Oscillation Workshop (NOW2018)

9 - 16 September, 2018

Rosa Marina (Ostuni, Brindisi, Italy)

\section{${ }^{1}$ Speaker}




\section{Introduction}

In the global context of the future experimental investigations of neutrino oscillation phenomenon, the JUNO detector [1] will play a central role on two aspects: the determination of mass hierarchy and the precise measurements of the solar oscillation parameters, i.e. $\Delta m_{21}^{2}$, $\sin ^{2} \theta_{12}$, as well as of the atmospheric squared mass difference $\Delta m_{31}^{2}$.

JUNO will be designed and realized as a huge liquid scintillator detector, therefore exploiting a mature and well proved technology, which has already provided fundamental contributions to the neutrino oscillation study through several implementations (Borexino [2], KamLAND [3], Daya Bay [4], Reno [5] and Double Chooz [6] being the most recent examples). It will base its measurements on the detection of the antineutrino flux coming from the cores of two nearby nuclear complexes, Yangjiang and Taishan, located at about $53 \mathrm{~km}$ from the experimental site.

The program will be complemented by a suite of astroparticle physics measurements which will significantly enhance the physics potential of JUNO, however not described here.

Overall goals, requirements, technical features and present status of the experiment are illustrated in the following.

\section{Summary of characteristics and of physics goals}

In Fig. 1 there is the summary of reactors' results accumulated so far, expressed as ratio of observed over expected events, compared with the prediction from the oscillation survival probability function. On the horizontal axis the reactor-detector distance is displayed; the plot reports the well-known circumstance that at small distance the impact of the oscillation phenomenon on the detector count rate is not visible, while it starts to manifest from roughly little less than $1 \mathrm{~km}$ baseline. At the special distance of $53 \mathrm{~km}$ the count rate suppression, mainly driven by the solar oscillation parameters, is maximal, therefore creating the best condition to study the interference effect governed in turn by the atmospheric mass squared difference, which is responsible for the ripple superimposed on the count rate suppressed profile. This is, therefore, the rationality beyond the choice of the optimum site and distance between JUNO and the emitting anti-neutrino cores.

To fully exploit this optimal baseline to perform an effective and successful measurement of the mass hierarchy, the detector must be endowed with two essential characteristics: large mass to perform a high statistic measurements, and stringent energy resolution to clearly distinguish the ripple induced by the atmospheric mass squared term. The two key numbers in this respects are the total mass of 20 kton of liquid scintillator, and the energy resolution of 3\% at $1 \mathrm{MeV}$, which represent, therefore, the major technical features characterizing the experiment.

In term of physics reach, such a high mass detector can tackle a plurality of measurements. Beyond mass hierarchy and precision determination of neutrino oscillation parameters, it can provide fundamental results concerning many hot topics in the astroparticle field, like supernova burst neutrinos, diffuse supernova neutrinos, solar neutrinos, atmospheric neutrinos, geo- 
neutrinos, sterile neutrinos, nucleon decay, indirect dark matter search, as well as a number of additional exotic searches; for details see the experiment's yellow book [7].

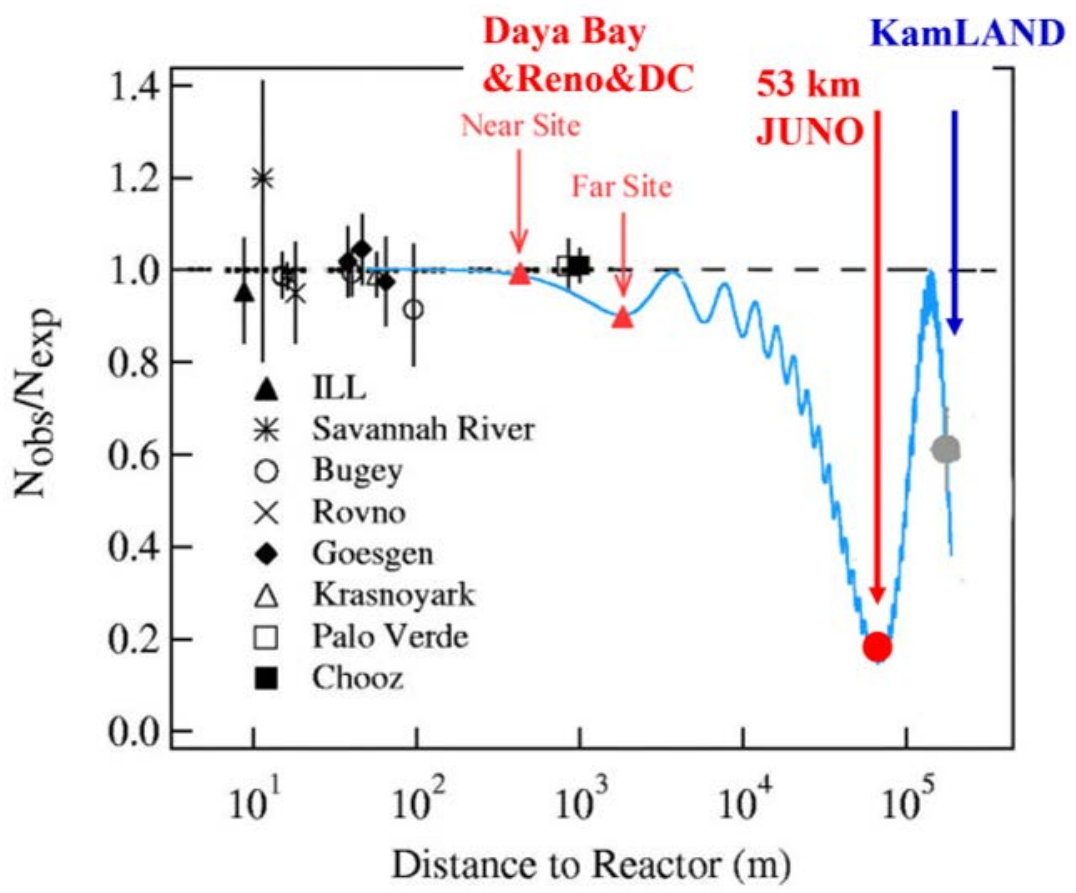

Fig. 1 - Summary of past reactors' results as ratio of observed to expected count rate, together with the predicted JUNO point

\section{Basic features of the program: detector structure, location and Collaboration}

JUNO is a spherical unsegmented liquid scintillator detector that will push such a technology beyond the present limit, as far as the mass (20 kton) and the resolution (3\%) are concerned. Succinctly, the detector (see Fig. 2) can be described as a large spherical acrylic vessel, which will hold the scintillator volume, contained in turn in a water pool, to ensure adequate shielding against the gamma radiation and neutrons from the rock.

The vessel will be surrounded by a stainless steel truss, which will perform the twofold task to sustain the vessel, by relieving its internal stress, and to provide the anchor support for the 18000 20" photomultipliers observing the scintillation photons. The light detection system will comprise also an additional set of 3" PMTs, up to 25000, which will be used for calibration purpose and to cross check the performances of the main PMTs, with the scope to control and reduce the systematic effects of the measurements performed by the main 20" PMT system.

Moreover, the shielding water around the acrylic vessel will be converted into a Cherenkov detector, being instrumented with about 2000 phototubes, which will detect the muon induced Cherenkov light. Such an arrangement, together with the top tracker that will be deployed on the roof of the detector itself, will allow an efficient muon veto capability, an essential feature at the planned shallow depth of the experiment, i.e. about $700 \mathrm{~m}$. 
JUNO has been approved in China at the beginning of 2013 and has been later joined by groups from all over the world. Currently the Collaboration encompasses 77 institutions from Asia, Europe and America, with more than 600 researchers, and it is still expanding.

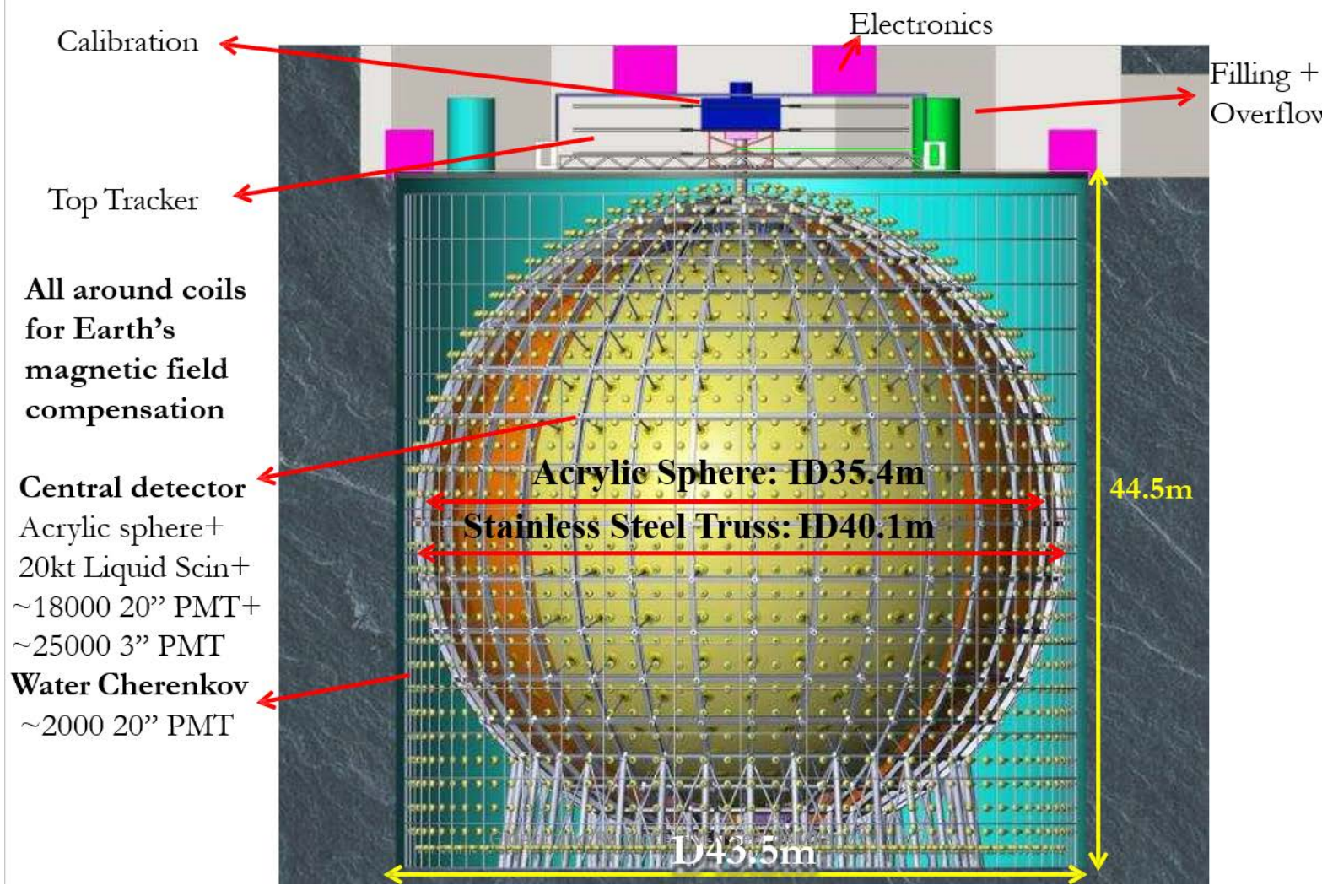

Fig. 2 - Schematic view of the JUNO configuration

The experiment is located in the South of China, Guangdong province, Jianmeng County, Kaiping city, at $53 \mathrm{~km}$ from the two sites of Yangjian and Taishan, where 6 and 4 nuclear cores are planned, respectively. By 2020 according to the construction schedule of the plants 26.6 GW will be installed ( 2 cores will be missing at Taishan), while eventually the total power of 35.8 GW will be available.

\section{How to infer the mass hierarchy}

The Inverse Beta Decay Reaction a là Cowan Reines used to detect the anti-neutrino signal is the following

$$
\bar{v}_{e}+p \rightarrow e^{+}+n
$$

The energy deposited by the positron in the scintillator, i.e. its kinetic energy plus the total $1.022 \mathrm{keV}$ energy of the two annihilation gammas, reflects faithfully the energy of the incoming anti-neutrinos

$$
E_{v i s}\left(e^{+}\right)=E(v)-0.8 \mathrm{MeV}
$$


$E_{v i s}\left(e^{+}\right)$is, thus, the specific measurement output to be analyzed for the hierarchy evaluation.

Through suitable approximations the survival probability Pee can be written as

$$
\begin{aligned}
P_{e e}=1-\cos ^{4} \theta_{13} \sin ^{2} 2 \theta_{12} \sin ^{2}\left(\Delta_{21}\right)-\sin ^{2} \theta_{13} \sin ^{2}\left(\left|\Delta_{31}\right|\right) & \\
& -\sin ^{2} \theta_{12} \sin ^{2} 2 \theta_{13} \sin ^{2}\left(\Delta_{21}\right) \cos \left(2\left|\Delta_{31}\right|\right) \\
\pm & \frac{\sin ^{2} \theta_{12}}{2} \sin ^{2} 2 \theta_{13} \sin \left(2 \Delta_{21}\right) \sin \left(2\left|\Delta_{31}\right|\right)
\end{aligned}
$$

In this expression, the phase $\Delta$ is obtained from the corresponding $\Delta \mathrm{m}^{2}$ by multiplication with the factor $\mathrm{L} / 4 \mathrm{E}$.

The sign flip in front of the last term is due to the hierarchy: positive for direct hierarchy, negative for the inverse one. The presence of the multiplicative factor $\sin ^{2} 2 \theta_{13}$ in this term questioned the effectiveness of this methodology, proposed for the first time in [8], until the experimental determination of the $\theta_{13}$ by Daya Bay, Reno and Double Chooz. Indeed, should $\theta_{13}$ have been resulted close to 0 , the last term of the Pee expression would have been vanishing small, making the proposed approach unfeasible. In reality, the discovery that $\theta_{13}$ is actually very close to the previous Chooz limit [9], opened the door to the actual implementation of the method.

The effect of Pee on the reactor spectrum is shown in Fig. 3; the y axis is proportional to the event rate, while on the $\mathrm{x}$ axis the ratio L/E_v is reported. The dashed line is the unoscillated spectrum; the continuous black line is the spectrum distorted and suppressed as an effect of the "solar" oscillation: this large effect is the key for the very precise determination of the two "solar" mixing parameters $\Delta m_{21}^{2}$ and $\sin ^{2} \theta_{12}$.

The blue and red lines superimposed on the smooth black line, instead, display the effect of the interference term driven by the atmospheric mass squared difference. The frequency of the ripple depends on $\left|\Delta m_{31}^{2}\right|$ (which therefore can also be determined with high accuracy from the precise "tracking" of the ripple itself), while its phase is linked to the $\mathrm{MH}$, as shown by the reciprocal shift of the blue and red lines in the figure. Unraveling the phase of the ripple, hence, is the clue for the MH determination.

To this purpose, obviously, the ripples must be preserved as much as possible throughout the detection process, setting the stringent requirement on the energy resolution of being equal or better than $3 \%$, representing by far the greatest challenge of the experiment.

Concrete $\chi^{2}$ calculations performed with the input parameters related to JUNO detector and site (i.e. baseline $53 \mathrm{~km}$, fiducial volume $20 \mathrm{kt}$, thermal reactor power $36 \mathrm{GW}$, exposure time 6 years, proton content 12\%, energy resolution 3\%) indicate that the statistical discrimination power of the experiment amounts to a $\Delta \chi^{2}$ equal to 16 between the true and wrong hierarchy hypothesis.

However, if systematic effects are considered, there is unavoidably a loss of discrimination power. The most important effect in this sense is the non-exactly equal baselines from the nuclear cores to the experiment, characterized by a spread of about $500 \mathrm{~m}$, which is responsible for a loss of 4 of the $\Delta \chi^{2}$ indicator. Other adverse effects detrimental to the 
discovery power are the $1 \%$ shape uncertainty of the reactor spectrum, and the background uncertainty (rate $4.5 \%$, shape $0.35 \%$ ). All in all, these effects bring the discrimination power down to $\Delta \chi^{2}=10.4$. Not considered here is the effect of the non-linearity of the energy scale, which through extensive calibrations is assumed will be kept well below $1 \%$, and thus almost irrelevant.

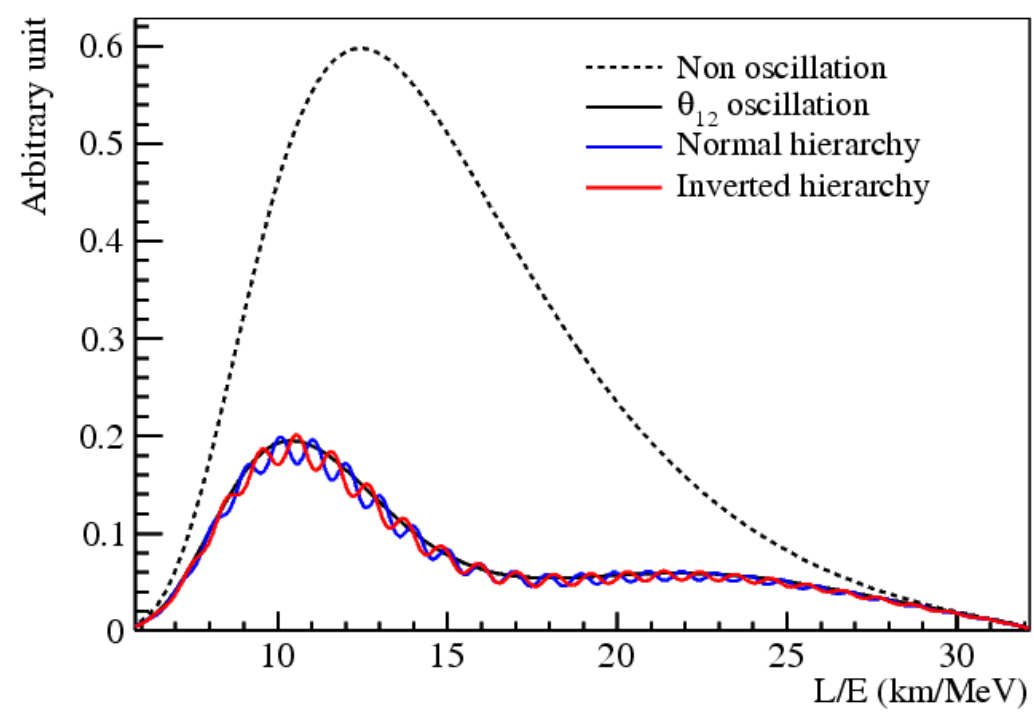

Fig. 3 -Effect of $P_{e e}$ electron neutrino survival probability on the reactor spectrum

On the other hand, by including in the analysis a penalty on the effective mass square difference, $\Delta m_{\mu \mu}^{2}$, as defined in [10], at the level of $1 \%$, anticipated as the precision available from the LBL experiments at the time of the JUNO data release, the discovery power of the experiment is brought back to the $\Delta \chi^{2}=16$ realm.

Another effect recently highlighted is the possible impact on the MH measurement of the sharp irregularities in the original reactor spectrum discussed in [11] (whose actual threat has been anyway debunked in [12]). To mitigate their influences, the Collaboration has decided to build a scintillator-based near detector, which will provide a high statistics and high resolution (about 1\%) assessment of the initial spectrum.

\section{Precision measurement of oscillation parameters}

The huge effect of the survival probability on the reactor spectrum and the large amount of data that will be accumulated (JUNO plans to record 100000 events in 6 years of data taking) make it possible to measure three of the mass-mixing parameters with unprecedented subpercent precision: the two solar parameters $\Delta m_{21}^{2}$ and $\sin ^{2} \theta_{12}$, and the effective parameter $\Delta m_{e e}^{2}$ defined as (see [10]) $\Delta m_{e e}^{2}=\cos ^{2} \theta_{12} \Delta m_{31}^{2}+\sin ^{2} \theta_{12} \Delta m_{32}^{2}$.

From pure statistical considerations, the uncertainty of the measurements of these parameters is predicted to be very limited, i.e. $0.54 \%, 0.24 \%$ and $0.27 \%$ for $\sin ^{2} \theta_{12}, \Delta m_{21}^{2}$ and $\Delta m_{e e}^{2}$, respectively, taking also into account the correlation among them. Even adding 
background and several systematic effects, like the spectrum bin to bin uncertainty, $1 \%$, the uncertainty on the absolute energy scale, $1 \%$, and the energy non linearity, again $1 \%$, the overall errors on the parameters remain below the $1 \%$ target: $0.67 \%$ for $\sin ^{2} \theta_{12}, 0.59 \%$ for $\Delta m_{21}^{2}$, and $0.44 \%$ for $\Delta m_{e e}^{2}$.

\section{JUNO progress and schedule}

The experiment is expected to start data taking at the beginning of the next decade. The ground breaking signaling the startup of the excavation occurred in January 2015. So far, the slope tunnel (1266 m) and the vertical shaft (564 m) have been fully excavated. The former will allow to bring the scintillator underground, the latter will enable access of personnel and construction materials. The excavation is now progressing with the digging of the experimental hall.

The civil construction is foreseen to be completed by about the end of 2019. The preparation of the detector components, e.g. phototubes, acrylic panels, etc., has already started in 2016, while the global onsite installation will be completed by the beginning of 2021. This scenario is in line to ensure the completion of the scintillator fill and the startup of data taking at the beginning of the next decade, before the end of 2021.

\section{Acknowledgments}

The author wishes to thank the organizers for the invitation to contribute to such an interesting and enlightening conference.

\section{References}

[1] T. Adam et al. (JUNO Collaboration), "The JUNO Conceptual Design Report", arXiv:1508.07166 (2016)

[2] G. Alimonti et al. (Borexino Collaboration), Nucl. Instr. and Meth and Meth. A 600, 568 (2009)

[3] S. Abe et al (KamLAND Collaboration), Phys. Rev. Lett. 100, 221803 (2008)

[4] F. P. An et al. (Daya Bay Collaboration), Phys. Rev. Lett. 108, 171803 (2012)

[5] J. K. Ahn et al., (RENO Collaboration), Phys. Rev. Lett. 108, 191802 (2012)

[6] S. Abe et al., (DOUBLE CHOOZ Collaboration), Phys. Lett. B, 723, 66 (2013)

[7] F. An et al. (JUNO Collaboration), J. of Phys. G (Nucl. and Part. Phys.) 43, id. 030401 (2016)

[8] S.T. Petcov and M. Piai, Physics Letters B 553, 94-106 (2002)

[9] M. Apollonio et al. (Chooz Collaboration), The European Physical Journal C 27, 331-374 (2003)

[10] H. Nunokawa, S. Parke, R. Zukanovich Funchal, Phys.Rev.D72, 013009 (2005)

[11] D.V. Forero et al., arXiv:1710.07378 (2017)

[12] Danielson et al., arXiv.1808.03276 (2018) 